\title{
A Compact Dual-Band Negative Group Delay Microwave Circuit
}

\author{
Te SHAO, Shaojun FANG, Zhongbao WANG, Hongmei LIU \\ School of Information Science and Technology, Dalian Maritime University, Dalian, Liaoning 116026, China \\ shortt@dlmu.edu.cn, fangshj@dlmu.edu.cn,wangzb@dlmu.edu.cn, lhm323@dlmu.edu.cn
}

Submitted May 18, 2018 / Accepted August 24, 2018

\begin{abstract}
A compact dual-band negative group delay circuit (NGDC) is proposed. The proposed NGDC is composed of an open-circuited transmission line and two resistors connected by two transmission lines. The frequency ratio is controlled by the characteristic impedance of the transmission lines. To verify the design concept, a dualband NGDC with the frequency ratio of $n=2$ (Circuit A) and a broadband NGDC with $n=1.16$ (Circuit B) are designed and fabricated. The measured group delay value of the Circuit $A$ is $-1.19 \mathrm{~ns}$ at the center frequencies of lower and upper bands. And the measured NGD bandwidth is $34.6 \%$ for the lower band and 16.5\% for the upper band, in which the return loss and insertion loss are better than $16.9 \mathrm{~dB}$ and $18.2 \mathrm{~dB}$, respectively. From the measurement results of Circuit $B$, a flat fractional NGD bandwidth of $19.8 \%$ with GD of $(-1.58 \pm 0.13)$ ns is obtained, in which the return loss and insertion loss are better than $23 \mathrm{~dB}$ and $32.5 \mathrm{~dB}$, respectively.
\end{abstract}

\section{Keywords}

Dual-band, broadband, negative group delay, compact, frequency ratio

\section{Introduction}

With the growing requirements of wireless communication systems, multiple communication standards have been developed. Therefore, various multi-band or broadband microwave components that can simultaneously support the different communication standards are necessary [1], [2]. Moreover, the group delay characteristics of circuits have drawn more attention for the reason that the group delay variations would cause signal distortion in the high-speed broadband communications [3]. To compensate for the group delay variations, delay equalizers based on negative group delay circuit (NGDC) have been adopted [4-6]. Because of their unique characteristic, the NGDCs have been used to increase the efficiency of the feed-forward amplifiers [7], enhance the bandwidth of the analog feed-back amplifiers [8], minimize the beam-squint in the array antennas [9], and realize the non-Foster elements [10].
The conventional NGDCs are usually implemented by RLC resonators [6-10]. In recent years, some new methods to obtain the NGD effect have been presented, such as feedback loop technique [11], [12], finite unloaded qualityfactor resonators [13], and signal interference techniques [14]. In addition, to obtain port matching without external matching networks, matched NGDCs using the coupled line doublet structure [15] and self-matched NGDCs [16] have been discussed. However, all of these NGDCs only operate in one frequency band.

To obtain dual-band or broadband NGDCs, a general approach that combines two or multi-NGDCs with different operating frequencies is adopted [17-19]. A four-stage circuit is utilized to achieve broadband NGDC [17], [18] and a two-stage FET-based active circuit is employed to realize dual-band NGDC [19]. In addition, some broadband NGDCs based on the transversal filter approach and distributed amplifiers are presented [20], [21], however, these approaches demand multi-stage structures. It is clear that the multi-stage structure certainly will increase the size of circuits. A miniaturized dual-band NGDC using dual-plane defected structures is proposed in [22]. Its center frequencies of the lower and upper bands are controlled by a defected microstrip structure (DMS) and a defected ground structure (DGS), respectively. Another dual-band NGDC is achieved by utilizing a couple of open-circuited stubs [23], whose center frequencies of the lower and upper bands are controlled by the lengths of stubs. However, these two kinds of NGDCs cannot obtain port matching. A design for a dual-band NGDC employing the composite right/lefthanded $\lambda / 4$ short stubs is discussed in [24]. But this NGDC is working as reflection-type that necessarily demands a broadband hybrid coupler to convert this circuit to the transmission-type circuit and realize port matching. Obviously, the use of extra coupler will increase the circuit size.

In this paper, a compact dual-band NGDC is proposed and self-matched without the need for external matching networks. The proposed NGDC is composed of an opencircuited transmission line and two resistors connected by two transmission lines. The frequency ratio is controlled by the characteristic impedance of the transmission lines. The theoretical analysis of the proposed NGDC is derived. The simulated and measured results are given and discussed. 


\section{Theory Analysis of Proposed NGDC}

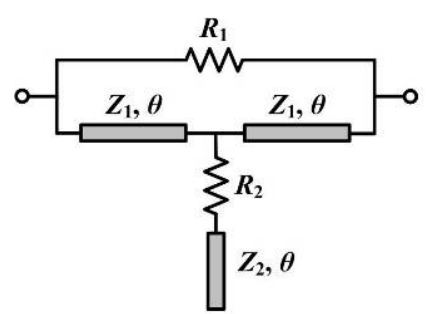

(a)
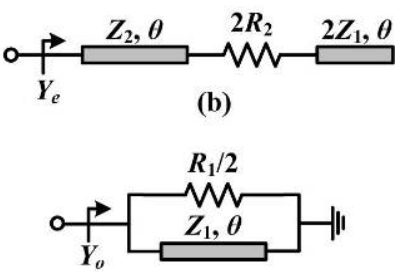

(c)
Fig. 1. (a) Schematic of the proposed NGDC. (b) Even-mode equivalent circuit. (c) Odd-mode equivalent circuit.

Figure 1(a) shows the structure of the proposed dualband NGDC. It consists of two resistors $R_{1}$ and $R_{2}$ connected by two transmission lines with characteristic impedance $Z_{1}$ and electrical length $\theta$, and an open-circuited transmission line with characteristic impedance $Z_{2}$ and electrical length $\theta$. The even- and odd-mode equivalent circuits are shown in Fig. 1(b) and (c), respectively. The even- and odd-mode input admittances $Y_{\mathrm{e}}$ and $Y_{\mathrm{o}}$ are respectively derived as

$$
\begin{gathered}
Y_{\mathrm{e}}=\frac{Z_{1}+2 Z_{2}+\mathrm{j} 2 R_{2} \tan \theta}{Z_{1}\left[2 R_{2}+\mathrm{j}\left(Z_{1} \tan \theta-2 Z_{2} \cot \theta\right)\right]}, \\
Y_{\mathrm{o}}=\frac{2}{R_{1}}+\frac{1}{\mathrm{j} Z_{1} \tan \theta}
\end{gathered}
$$

where $\theta=\pi f /\left(2 f_{0}\right)$ and $f_{0}=\left(f_{1}+f_{2}\right) / 2 . f_{1}$ and $f_{2}$ are the center frequencies of the lower and upper bands, respectively. Then the $S$-parameters of the dual-band NGDC can be derived as

$$
\begin{aligned}
& S_{11}=S_{22}=\frac{Y_{0}^{2}-Y_{\mathrm{e}} Y_{\mathrm{o}}}{\left(Y_{0}+Y_{\mathrm{e}}\right)\left(Y_{0}+Y_{\mathrm{o}}\right)}, \\
& S_{12}=S_{21}=\frac{Y_{0}\left(Y_{\mathrm{o}}-Y_{\mathrm{e}}\right)}{\left(Y_{0}+Y_{\mathrm{e}}\right)\left(Y_{0}+Y_{\mathrm{o}}\right)}
\end{aligned}
$$

where $Y_{0}=1 / Z_{0}$ and $Z_{0}$ is the port impedance. Substituting (1) and (2) into (4), $S_{21}$ can be obtained as

$$
S_{21}=\frac{X_{1}+\mathrm{j} X_{2}}{X_{3}+\mathrm{j} X_{4}}
$$

Thus the group delay (GD) of the proposed circuit can be obtained as

$$
\tau=-\frac{\mathrm{d} \angle S_{21}}{\mathrm{~d} \omega}=\frac{X_{1}^{\prime} X_{2}-X_{1} X_{2}^{\prime}}{X_{1}^{2}+X_{2}^{2}}-\frac{X_{3}^{\prime} X_{4}-X_{3} X_{4}^{\prime}}{X_{3}^{2}+X_{4}^{2}}
$$

The values of $X_{1}, X_{2}, X_{3}, X_{4}, X_{1}^{\prime}, X_{2}^{\prime}, X_{3}^{\prime}$, and $X_{4}^{\prime}$ are given as

$$
\begin{gathered}
X_{1}=Y_{0}\left(2 / R_{1}-M\right), \\
X_{2}=-Y_{0}\left[N+1 /\left(Z_{1} \tan \theta\right)\right],
\end{gathered}
$$

$$
\begin{aligned}
& X_{3}=\left(Y_{0}+M\right)\left(Y_{0}+2 / R_{1}\right)+N /\left(Z_{1} \tan \theta\right), \\
& X_{4}=N\left(Y_{0}+2 / R_{1}\right)-\left(Y_{0}+M\right) /\left(Z_{1} \tan \theta\right), \\
& X_{1}^{\prime}=-Y_{0} M^{\prime}, \\
& X_{2}^{\prime}=Y_{0}\left[1 /\left(4 f_{0} Z_{1} \sin ^{2} \theta\right)-N^{\prime}\right] \\
& X_{3}^{\prime}=M^{\prime}\left(Y_{0}+2 / R_{1}\right)+N^{\prime} /\left(Z_{1} \tan \theta\right)-N /\left(4 f_{0} Z_{1} \sin ^{2} \theta\right) \text {, } \\
& X_{4}^{\prime}=\left(Y_{0}+2 / R_{1}\right) N^{\prime}-M^{\prime} /\left(Z_{1} \tan \theta\right) \\
& +\left(Y_{0}+M\right) /\left(4 f_{0} Z_{1} \sin ^{2} \theta\right) \\
& M=2 R_{2}\left(1+\tan ^{2} \theta\right) / P \\
& N=Q /\left(Z_{1} P\right) \text {, } \\
& P=4 R_{2}^{2}+\left(Z_{1} \tan \theta-2 Z_{2} \cot \theta\right)^{2}, \\
& Q=4 R_{2}^{2} \tan \theta-\left(Z_{1}+2 Z_{2}\right)\left(Z_{1} \tan \theta-2 Z_{2} \cot \theta\right) \\
& M^{\prime}=\left[R_{2} \tan \theta \cdot P /\left(f_{0} \cos ^{2} \theta\right)-2 R_{2}\left(1+\tan ^{2} \theta\right) P^{\prime}\right] / P^{2}, \\
& N^{\prime}=\left(P Q^{\prime}-P^{\prime} Q\right) /\left(Z_{1} P^{2}\right), \\
& P^{\prime}=2\left(Z_{1} \tan \theta-2 Z_{2} \cot \theta\right)\left(\frac{Z_{1}}{4 f_{0} \cos ^{2} \theta}+\frac{2 Z_{2}}{4 f_{0} \sin ^{2} \theta}\right), \\
& Q^{\prime}=\frac{R_{2}^{2}}{f_{0} \cos ^{2} \theta}-\left(Z_{1}+2 Z_{2}\right)\left(\frac{Z_{1}}{4 f_{0} \cos ^{2} \theta}+\frac{Z_{2}}{2 f_{0} \sin ^{2} \theta}\right) \\
& -\left(Z_{1} \tan \theta-2 Z_{2} \cot \theta\right)\left(\frac{Z_{2} \cot \theta}{2 f_{0} \cos ^{2} \theta}-\frac{Z_{2} \tan \theta}{2 f_{0} \sin ^{2} \theta}\right) \text {. }
\end{aligned}
$$

According to the periodicity of the trigonometric functions, the relations can be obtained as

$$
\left\{\begin{array}{c}
X_{i}(\theta)=X_{i}(\pi-\theta) \\
X_{j}(\theta)=-X_{j}(\pi-\theta) \\
X_{i}^{\prime}(\theta)=-X_{i}^{\prime}(\pi-\theta) \\
X_{j}^{\prime}(\theta)=X_{j}^{\prime}(\pi-\theta)
\end{array}, i=1,3 ; j=2,4 .\right.
$$

The GD can then be derived as

$$
\tau(\theta)=\tau(\pi-\theta) \text {. }
$$

Thus the proposed NGDC can get the same GD at the electrical length $\theta_{1}$ and $\theta_{2}=\pi-\theta_{1}$. And the desired frequencies $f_{1}$ and $f_{2}$ satisfy the relations as

$$
n=\frac{f_{2}}{f_{1}}=\frac{\theta_{2}}{\theta_{1}}=\frac{\pi-\theta_{1}}{\theta_{1}},
$$




$$
\begin{aligned}
& f_{1}=\frac{2 f_{0}}{1+n}, \\
& f_{2}=\frac{2 n f_{0}}{1+n} .
\end{aligned}
$$

In theory, the proposed NGDC can operate in two arbitrary frequency bands, but in practice, the construction of the NGDC is also constrained by the range of impedance that can be realized. The required $Z_{1}$ for different frequency ratios is shown in Fig. 2. The required $Z_{1}$ increases as the frequency ratio increases, and the $Z_{1}$ increases as the $Z_{2}$ increases for the fixed frequency ratio. The calculated values of GD $\times f_{0}$ as a function of $Z_{1}$ with different $Z_{2}$ are given in Fig. 3. The absolute value of NGD time increases as $Z_{2}$ increases, and slightly increases as the $Z_{1}$ increases from $120 \Omega$ to $200 \Omega$. However, when the $Z_{1}$ is close to $100 \Omega$ corresponding to a frequency ratio close to 1 , the GD will be superimposed and the absolute value of NGD time will obviously increase. Therefore, the desired frequency ratio and GD can be obtained by choosing appropriate $Z_{1}$ and $Z_{2}$.

To realize the input and output matching, the condition that $S_{11}=S_{22}=0$ should be satisfied at the designed frequencies which leads to the relations as follows

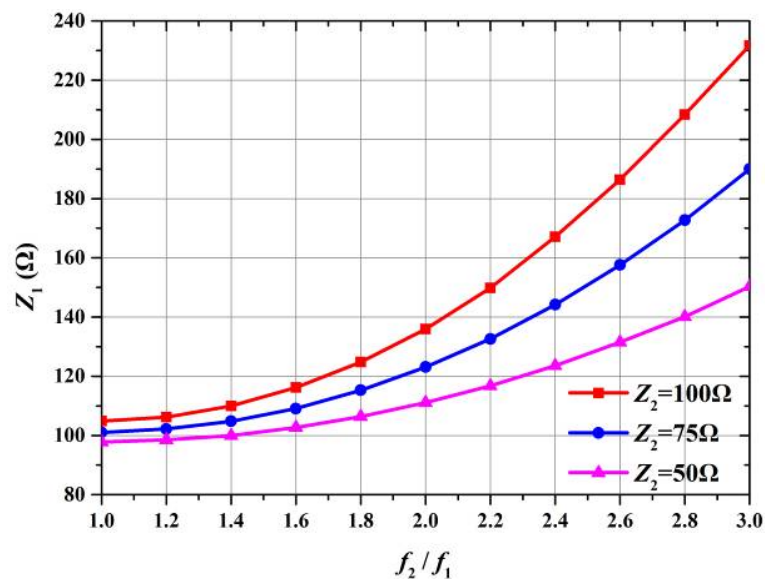

Fig. 2. Required $Z_{1}$ for different frequency ratios with different $Z_{2}$.

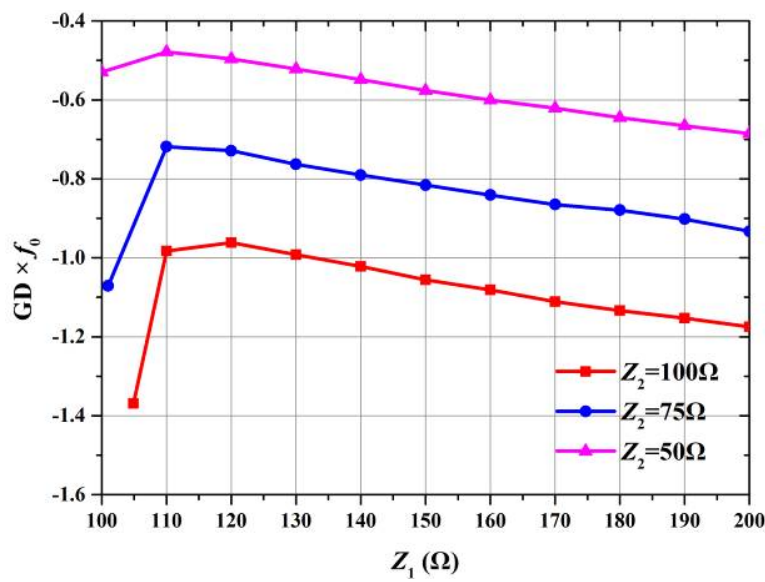

Fig. 3. Calculated values of GD $\times f_{0}$ as a function of $Z_{1}$ with different $Z_{2}$.

$$
\begin{gathered}
F\left(R_{2}\right)=Y_{0}^{2}-\left(M^{2}+N^{2}\right) /\left(N Z_{1} \tan \theta\right)=0, \\
R_{1}=\left(2 N Z_{1} \tan \theta\right) / M
\end{gathered}
$$

Thus the required $R_{1}$ and $R_{2}$ related to Fig. 3 can be calculated according to (28) and (29) and shown in Fig. 4. The required $R_{1}$ and $R_{2}$ decreases as $Z_{1}$ increases or $Z_{2}$ decreases. The $R_{1}$ and $R_{2}$ also influence the GD and frequency ratio. As shown in Fig. 5, the frequency ratio increases as $R_{1}$ and $R_{2}$ decrease, and the absolute value of GD time increases as $R_{1}$ increases while decreases as $R_{2}$ increases.

To achieve larger NGD time under the condition of fixed frequency, the appropriate combination of $R_{1}$ and $R_{2}$ should be chosen. Figure 6 gives the $S$-parameters and GD with different combinations of $R_{1}$ and $R_{2}$ with the frequency $f_{0}=1 \mathrm{GHz}, Z_{1}=136 \Omega$ and $Z_{2}=100 \Omega$. According to (28) and (29), the matching conditions at designed frequencies cannot always be satisfied when the values of $R_{1}$ and $R_{2}$ change. But as shown in Fig. 6, within the frequency ranges that GD is less than $0 \mathrm{~ns}$, the $\left|S_{11}\right|$ is still better than $-15 \mathrm{~dB}$
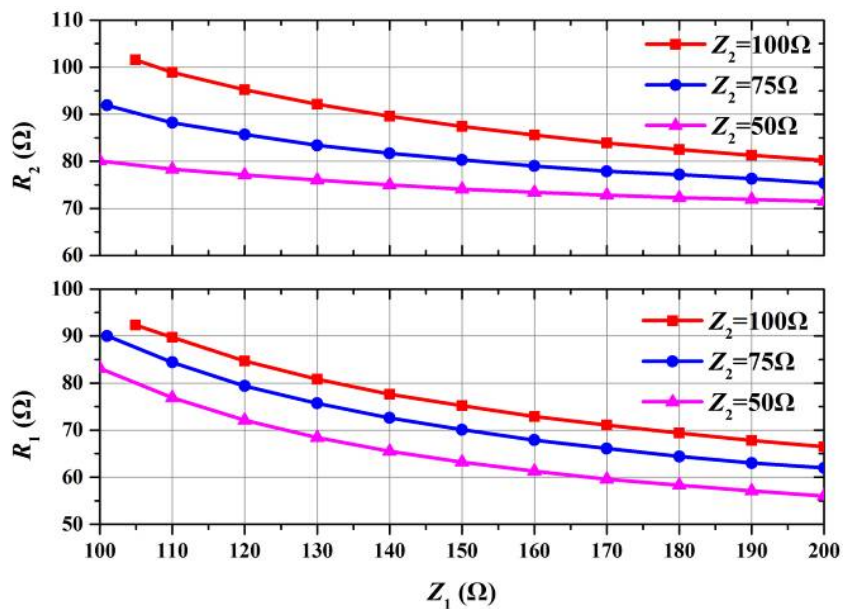

Fig. 4. Required $R_{1}$ and $R_{2}$ as a function of $Z_{1}$ with different $Z_{2}$.
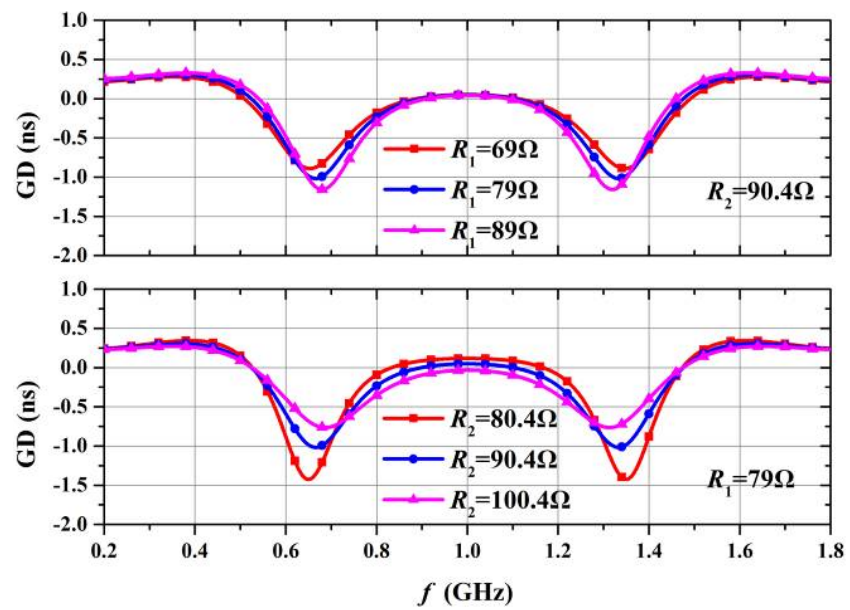

Fig. 5. Calculated GD with different $R_{1}$ and $R_{2}$ with $f_{0}=1 \mathrm{GHz}, Z_{1}=130 \Omega$ and $Z_{2}=100 \Omega$. 

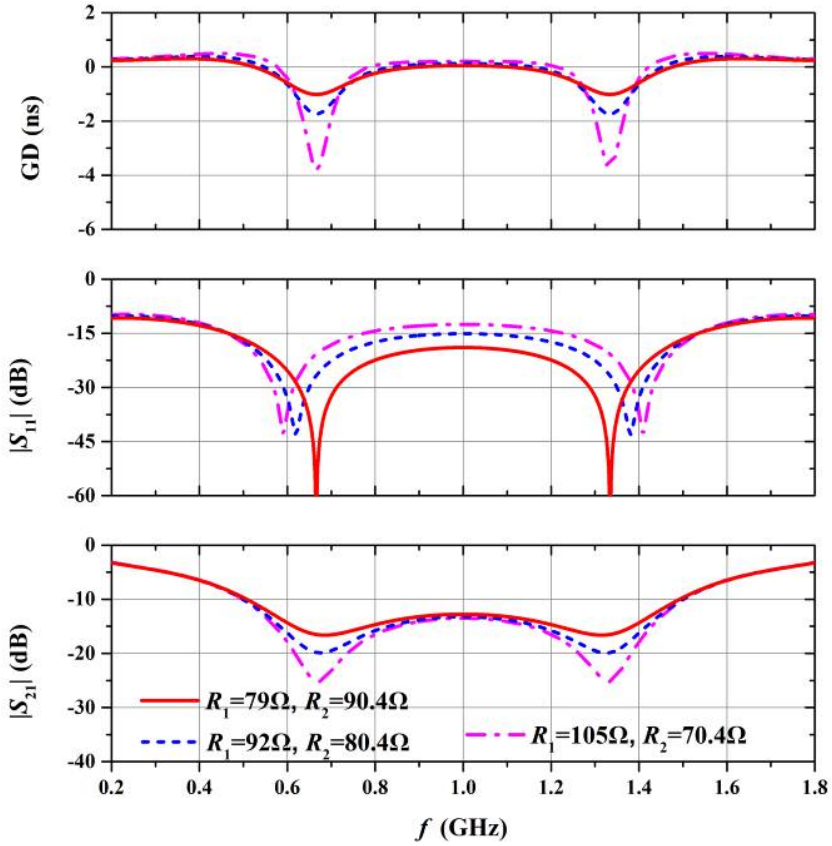

Fig. 6. Calculated $S$-parameters and GD with different combinations of $R_{1}$ and $R_{2}$ with $f_{0}=1 \mathrm{GHz}, Z_{1}=136 \Omega$ and $Z_{2}=100 \Omega$.

Based on the preceding analysis, the design procedures of the proposed dual-band NGDC are summarized as follows.

1) Determine the frequency ratio and NGD time according to the design requirements. Obtain the values of dielectric constant and thickness of the substrate material.

2) Select proper $Z_{1}$ and $Z_{2}$ according to the frequency ratio and NGD time requirements referring to Fig. 2 and 3.

3 ) Calculate $R_{1}$ and $R_{2}$ according to (28) and (29). If a larger NGD time is wanted, choose the appropriate combinations of $R_{1}$ and $R_{2}$ referring to Fig. 5 .

4) Convert all the electrical parameters to the physical dimensions and optimize the physical dimensions to obtain the correct NGD time and the required frequency ratio using a full-wave electromagnetic simulator ANSYS HFSS. If the proper physical dimensions with the required

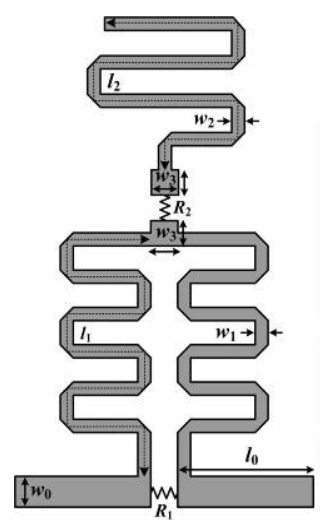

(a)

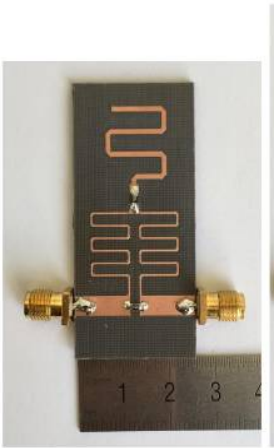

(b)

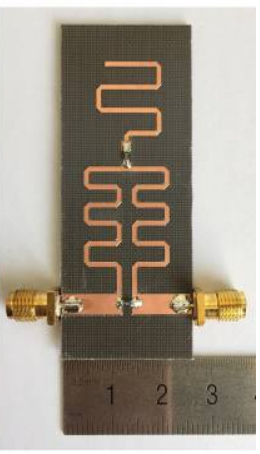

(c)
Fig. 7. Layout and photographs of the proposed NGDCs. (a) Layout. (b) Circuit A. (c) Circuit B. performance cannot be realized, the design should be restarted from the Step 2) with other potential values.

\section{Implementation and Performance}

To validate the design concept of the proposed NGDC, a dual-band NGDC with $n=2$ (Circuit A) and a broadband NGDC with $n=1.16$ (Circuit B) are designed with $f_{0}=1 \mathrm{GHz}$. The proposed NGDCs are fabricated on the substrate with relative permittivity $\varepsilon_{\mathrm{r}}=2.65$ and thickness $h=1.5 \mathrm{~mm}$. The transmission lines are meandered to reduce the circuit size as shown in Fig. 7.

\subsection{Design of a Dual-band NGDC}

As the first example, a dual-band NGDC with $n=2$ is designed. In this design, the electrical parameters are selected as $Z_{1}=136 \Omega, Z_{2}=100 \Omega, R_{1}=79 \Omega$ and $R_{2}=$ $90.4 \Omega$. Considering the actual value of resistors, the influence of connecting lines and the bend discontinuities of the microstrip line, the proposed NGDC is optimized by ANSYS HFSS. The optimized physical dimensions are $w_{0}=4 \mathrm{~mm}, \quad l_{0}=12 \mathrm{~mm}, \quad w_{1}=0.4 \mathrm{~mm}, \quad l_{1}=64.7 \mathrm{~mm}$, $w_{2}=1 \mathrm{~mm}, l_{2}=57.1 \mathrm{~mm}$ and $w_{3}=2 \mathrm{~mm}$ with $R_{1}=82 \Omega$ and $R_{2}=91 \Omega$. The photograph of the fabricated Circuit A is shown in Fig. 7(b) and the overall size of the circuit is

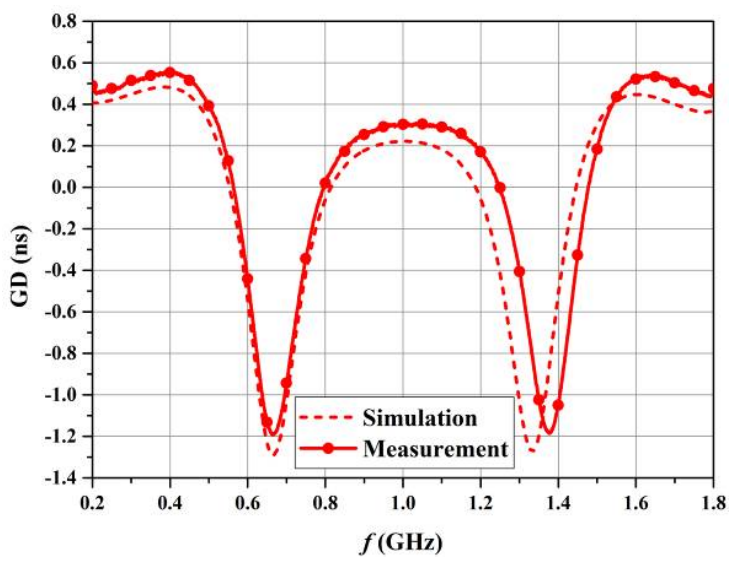

Fig. 8. Simulated and measured group delay of the designed Circuit A.

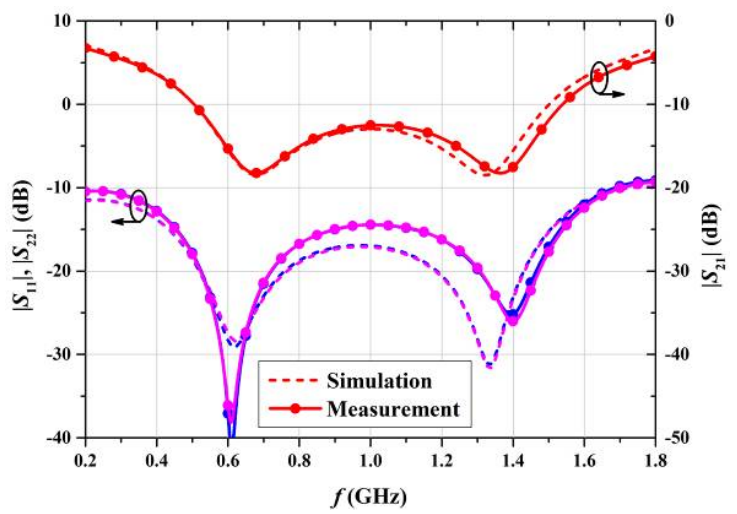

Fig. 9. Simulated and measured $S$-parameters of the designed Circuit A. 


\begin{tabular}{|c|c|c|c|c|c|c|c|c|c|c|c|c|c|}
\hline \multirow{2}{*}{ Ref } & \multicolumn{2}{|c|}{$f(\mathrm{GHz})$} & \multicolumn{2}{|c|}{ GD (ns) } & \multicolumn{2}{|c|}{ NGD FBW (\%) } & \multicolumn{2}{|c|}{ IL (dB) } & \multicolumn{2}{|c|}{$\mathrm{RL}$ (dB) } & \multicolumn{2}{|c|}{ FOM } & \multirow{2}{*}{$\begin{array}{l}\text { Circuit size } \\
\left(\lambda_{\mathrm{g}} \times \lambda_{\mathrm{g}}\right)\end{array}$} \\
\hline & $f_{1}$ & $f_{2}$ & $f_{1}$ & $f_{2}$ & $f_{1}$ & $f_{2}$ & $f_{1}$ & $f_{2}$ & $f_{1}$ & $f_{2}$ & $f_{1}$ & $f_{2}$ & \\
\hline [22] & 3.50 & 5.15 & -4.54 & -4.20 & 3.43 & 1.94 & 47.4 & 38.8 & - & - & 0.0023 & 0.0048 & $0.36 \times 0.83$ \\
\hline [23] & 3.50 & 5.20 & -5.00 & -5.00 & 5.71 & 5.77 & 13.0 & 19.5 & - & - & 0.2240 & 0.1590 & $0.60 \times 0.64$ \\
\hline [24] & 2.14 & 3.50 & -3.00 & -3.10 & 8.41 & 5.14 & 34.2 & 34.9 & 17 & 17 & 0.0105 & 0.0100 & $0.90 \times 1.80$ \\
\hline $\begin{array}{l}\text { This } \\
\text { work }\end{array}$ & 0.667 & 1.377 & -1.19 & -1.19 & 34.6 & 16.5 & 18.2 & 18.2 & 24.8 & 24.7 & 0.0338 & 0.0332 & $0.13 \times 0.27$ \\
\hline
\end{tabular}

Tab. 1. Performance comparison of the proposed Circuit A with other works.

\begin{tabular}{|c|c|c|c|c|c|c|c|}
\hline Ref & $\boldsymbol{f}_{\mathbf{0}}(\mathbf{G H z})$ & GD $(\mathbf{n s})$ & $\begin{array}{c}\text { NGD BW } \\
(\mathbf{M H z})\end{array}$ & $\begin{array}{c}\text { flat NGD BW } \\
(\mathbf{M H z})\end{array}$ & IL (dB) & RL (dB) & $\begin{array}{c}\text { Circuit size } \\
\left(\lambda_{g} \times \lambda_{g}\right)\end{array}$ \\
\hline$[20]$ & 1.010 & $-1.55 \pm 0.12$ & 370 & 125 & 33.0 & 25.0 & $0.06 \times 0.78$ \\
\hline$[21]$ & 0.865 & $-0.65 \pm 0.55$ & 620 & 530 & 10.0 & 20.0 & $0.48 \times 0.65$ \\
\hline $\begin{array}{c}\text { This } \\
\text { work }\end{array}$ & $\mathbf{0 . 9 9 8}$ & $\mathbf{- 1 . 5 8} \pm \mathbf{0 . 1 3}$ & $\mathbf{4 4 6}$ & $\mathbf{1 9 8}$ & $\mathbf{3 2 . 5}$ & $\mathbf{2 3 . 2}$ & $\mathbf{0 . 1 3} \times \mathbf{0 . 3 2}$ \\
\hline
\end{tabular}

Tab. 2. Performance comparison of the proposed Circuit B with other works.

$55 \mathrm{~mm} \times 26 \mathrm{~mm}$ (around $0.27 \lambda_{\mathrm{g}} \times 0.13 \lambda_{\mathrm{g}}$, where $\lambda_{\mathrm{g}}$ is the guided wavelength of $50-\Omega$ TLs at the frequency $f_{0}$ ). The fabricated prototype was measured with an Agilent N5230A network analyzer.

Figures 8 and 9 give the simulated and measured group delay and $S$-parameters of the Circuit A. For the lower band, the measured GD and insertion loss (IL) at $f_{1}=0.667 \mathrm{GHz}$ are $-1.19 \mathrm{~ns}$ and $18.2 \mathrm{~dB}$, respectively. For the upper band, the same GD and IL are obtained at $f_{2}=1.377 \mathrm{GHz}$. The frequency ratio of the measured minimum GD for dual-band is 2.06. The fractional NGD bandwidths (the bandwidth for GD less than $0 \mathrm{~ns}$ ) are $34.6 \%$ (564-795 MHz) for the lower band and 16.5\% (1250 to $1477 \mathrm{MHz}$ ) for the upper band, respectively. Within both NGD bands, the return losses (RL) are better than $16.9 \mathrm{~dB}$. There are some discrepancies between the simulated and measured results, which is mainly due to the inaccurate value of the chip resistors used in the fabricated prototype.

Table 1 compares the proposed Circuit A with previous publications. The proposed dual-band NGDC has larger NGD bandwidth and better return losses performance than that in [22-24]. Except for [23], the proposed Circuit $\mathrm{A}$ has a better figure of merit (FOM) which can be defined as

$$
F O M=\left|\tau\left(f_{m}\right)\right| \cdot B W_{\mathrm{NGD}} \cdot\left|S_{21}\left(f_{m}\right)\right|, m=1,2 .
$$

In addition, the circuit size of the proposed Circuit $\mathrm{A}$ is smaller than all the previous works [22-24].

\subsection{Design of a Broadband NGDC}

In the second example, the electrical parameters of the broadband NGDC with $n=1.16$ are chosen as $Z_{1}=$ $105.7 \Omega, Z_{2}=100 \Omega, R_{1}=91.9 \Omega$ and $R_{2}=101 \Omega$. After optimization, the physical dimensions are $w_{0}=4 \mathrm{~mm}, l_{0}=$ $12 \mathrm{~mm}, w_{1}=1 \mathrm{~mm}, l_{1}=63.7 \mathrm{~mm}, w_{2}=1 \mathrm{~mm}, l_{2}=59.8 \mathrm{~mm}$ and $w_{3}=2 \mathrm{~mm}$ with $R_{1}=91 \Omega$ and $R_{2}=110 \Omega$. As shown

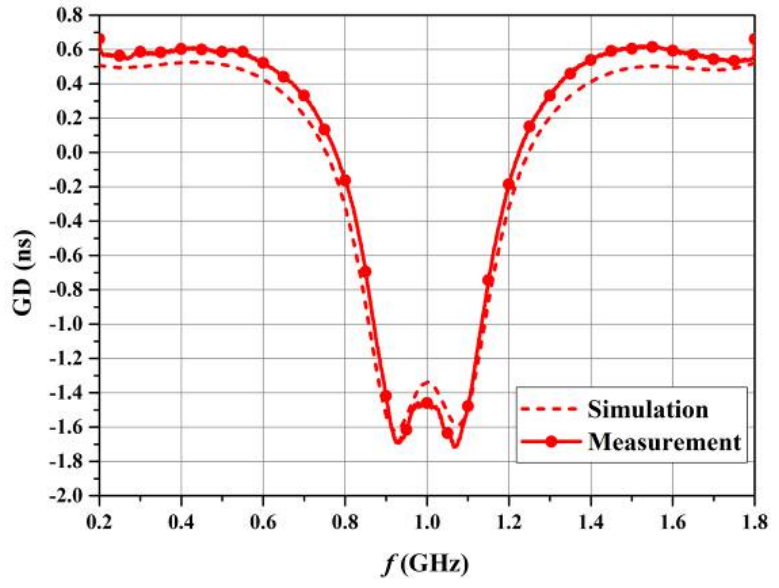

Fig. 10. Simulated and measured group delay of the designed Circuit B.

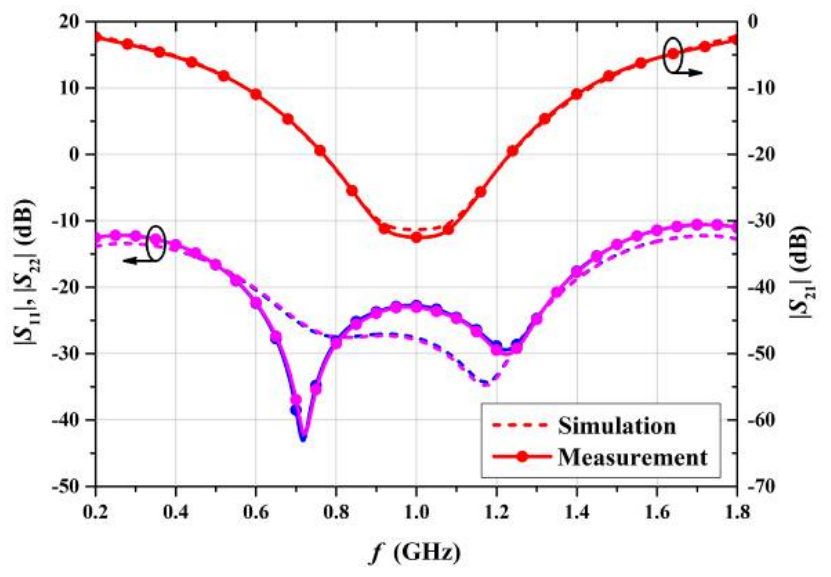

Fig. 11. Simulated and measured $S$-parameters of the designed Circuit B.

in Fig. 7(c), the overall size of the Circuit $B$ is $65 \mathrm{~mm} \times 26 \mathrm{~mm}$ (around $0.32 \lambda_{\mathrm{g}} \times 0.13 \lambda_{\mathrm{g}}$ ). The simulated and measured GD and $S$-parameters of the Circuit B are shown in Fig. 10 and 11. From the measurement, the NGD bandwidth is $446 \mathrm{MHz}$ and a flat NGD bandwidth of 
$198 \mathrm{MHz}$ with GD of $(-1.58 \pm 0.13) \mathrm{ns}$ is obtained. The IL and RL within the NGD band are better than $32.5 \mathrm{~dB}$ and $23 \mathrm{~dB}$, respectively.

Table 2 summarizes the comparison between the proposed Circuit B and other broadband NGDC. Compared to the NGDC in [20], the proposed Circuit B has the same level of GD, but provides a wider NGD bandwidth and a wider flat NGD bandwidth. The GD of the proposed Circuit B fluctuates less within the NGD band than that in [21], which demonstrates a better NGD flatness performance. In addition, the proposed Circuit B has smaller circuit size than the previous works [20], [21].

\section{Conclusion}

In this paper, a compact dual-band NGDC was proposed. The NGDC was implemented by two transmission lines, an open-circuited transmission line and two resistors. The theoretical analysis shows that arbitrary frequency ratio can be obtained by choosing appropriate characteristic impedances of transmission lines. Using the proposed method, two NGDCs with the frequency ratio of $n=2$ (Circuit A) and 1.16 (Circuit B) were implemented. The Circuit A demonstrated a dual-band performance and the Circuit B provided a broadband performance with a wide flat NGD bandwidth. The proposed NGDCs have smaller circuit size than the conventional ones achieved by using multi-stage structures. The proposed NGDCs can be used in various applications.

\section{Acknowledgments}

This work was supported jointly by the National Natural Science Foundation of China (No. 61871417, 61571075 and 51809030), the Youth Science and Technology Star Project Support Program of Dalian City (2016RQ038), the China Postdoctoral Science Foundation (No. 2017M611210), and the Fundamental Research Funds for the Central Universities (No. 3132018185 and 3132016320).

\section{References}

[1] MENG, X. M., YU, C. P., WU, Y. L., et al. Design of dual-band high-efficiency power amplifiers based on compact broadband matching networks. IEEE Microwave Wireless Components Letters, 2018, vol. 28, no. 2, p. 162-164. DOI: 10.1109/LMWC.2017.2787058

[2] LIU, Y., JIANG, S., ZHU, S., et al. Large frequency-ratio dualband and broad dual-band parallel-line couplers. IEEE Transactions on Components, Packaging and Manufacturing Technology, 2018, vol. 8, no. 1, p. 121-131. DOI: 10.1109/TCPMT.2017.2761991

[3] MYOUNG, S. S., KWON, B. S., KIM, Y. H., et al. Effect of group delay in RF BPF on impulse radio systems. IEICE Transactions on
Communications, 2007, vol. 90, no. 12, p. 3514-3522. DOI: 10.1093/ietcom/e90-b.12.3514

[4] EUDES, T., RAVELO, B. Cancellation of delays in the high-rate interconnects with UWB NGD active cells. Applied Physics Research, 2011, vol. 3, no. 2, p. 81-88. DOI: 10.5539/apr.v3n2p81

[5] AHN, K. P., ISHIKAWA, R., HONJO, K. Group delay equalized UWB InGaP/GaAs HBT MMIC amplifier using negative group delay circuits. IEEE Transactions on Microwave Theory and Techniques, 2009, vol. 57, no. 9, p. 2139-2147. DOI: 10.1109/TMTT.2009.2027082

[6] RAVELO, B., PERENNEC, A., ROY, M. L. Experimental validation of the $\mathrm{RC}$-interconnect effect equalization with negative group delay active circuit in planar hybrid technology. In IEEE Workshop on Signal Propagation on Interconnects. Strasbourg, (France), 2009, p. 1-4. DOI: 10.1109/SPI.2009.5089836

[7] CHOI, H., JEONG, Y., KIM, C. D., et al. Efficiency enhancement of feedforward amplifiers by employing a negative group-delay circuit. IEEE Transactions on Microwave Theory and Techniques, 2010, vol. 58, no. 5, p. 1116-1125. DOI: 10.1109/TMTT.2010.2045576

[8] CHOI, H., JEONG, Y., KIM, C. D., et al. Bandwidth enhancement of an analog feed-back amplifier by employing a negative group delay circuit. Progress in Electromagnetics Research, 2010, vol. 105 , p. 253-272. DOI: 10.2528/PIER10041808

[9] OH, S. S., SHAFAI, L. Compensated circuit with characteristics of lossless double negative materials and its application to array antennas. IET Microwaves Antennas and Propagation, 2007, vol. 1, no. 1, p. 29-38. DOI: 10.1049/iet-map:20050229

[10] MIRZAEI, H., ELEFTHERIADES, G. V. Realizing non-Foster reactive elements using negative-group-delay networks. IEEE Transactions on Microwave Theory and Techniques, 2013, vol. 61, no. 12, p. 4322-4332. DOI: 10.1109/TMTT.2013.2281967

[11] RAVELO, B. Theory of coupled line coupler-based negative group delay microwave circuit. IEEE Transactions on Microwave Theory and Techniques, 2016, vol. 64, no. 11, p. 3604-3611. DOI: 10.1109/TMTT.2016.2604316

[12] RAVELO, B. Innovative theory on multiband NGD topology based on feedback-loop power combiner. IEEE Transactions on Circuits and Systems II Express Briefs, 2016, vol. 63, no. 8, p. 738-742. DOI: 10.1109/TCSII.2016.2531101

[13] CHAUDHARY, G., JEONG, Y. A finite unloaded quality-factor resonators based negative group delay circuit and its application to design power divider. Microwave and Optical Technology Letters, 2016, vol. 58, no. 12 , p. 2918-2921. DOI: 10.1002/mop.30184

[14] WANG, Z., CAO, Y., SHAO, T., et al. A negative group delay microwave circuit based on signal interference techniques. IEEE Microwave Wireless Components Letters, 2018, vol. 28, no. 4, p. 290-292. DOI: 10.1109/LMWC.2018.2811254

[15] CHAUDHARY, G., JEONG, Y. Transmission-type negative group delay networks using coupled line doublet structure. IET Microwaves Antennas and Propagation, 2015, vol. 9, no. 8, p. 748-754. DOI: 10.1049 /iet-map.2014.0351

[16] SHAO, T., WANG, Z., FANG, S., et al. A compact transmissionline self-matched negative group delay microwave circuit. IEEE Access, 2017, vol. 5, p. 22836-22843. DOI: 10.1109/ACCESS.2017.2761890

[17] BROOMFIELD, C. D., EVERARD, J. K. A. Broadband negative group delay networks for compensation of microwave oscillators and filters. Electronics Letters, 2000, vol. 36, no. 23, p. 1931-1933. DOI: 10.1049/el:20001377

[18] RAVElo, B., PERENNEC, A., ROY, M. L. Synthesis of broadband negative group delay active circuits. In IEEE MTT-S International Microwave Symposium Digest. Honolulu (USA), 2007, p. 2177-2180. DOI: 10.1109/MWSYM.2007.380357 
[19] RAVELO, B., BLASI, S. D. An FET-based microwave active circuit with dual-band negative group delay. Journal of Microwaves, Optoelectronics and Electromagnetic Application, 2011, vol. 10, no. 2, p. 355-366. DOI: 10.1590/S2179-10742011000200006

[20] WU, C. T. M., ITOH, T. Maximally flat negative group-delay circuit: a microwave transversal filter approach. IEEE Transactions on Microwave Theory and Techniques, 2014, vol. 62, no. 6, p. 1330-1342. DOI: 10.1109/TMTT.2014.2320220

[21] WU, C. T. M., GHARAVI, S., DANESHRAD, B., et al. A dualpurpose reconfigurable negative group delay circuit based on distributed amplifiers. IEEE Microwave Wireless Components Letters, 2013, vol. 23, no. 11, p. 593-595. DOI: 10.1109/LMWC.2013.2279104

[22] CHAUDHARY, G., JEONG, Y., LIM, J. Miniaturized dual-band negative group delay circuit using dual-plane defected structures. IEEE Microwave Wireless Components Letters, 2014, vol. 24, no. 8, p. 521-523. DOI: 10.1109/LMWC.2014.2322445

[23] TAHER, H., FARRELL, R. Dual wide-band miniaturized negative group delay circuit using open circuit stubs. Microwave and Optical Technology Letters, 2018, vol. 60, no. 2, p. 428-432. DOI: 10.1002/mop.30979

[24] CHOI, H., JEONG, Y., LIM, J., et al. A novel design for a dualband negative group delay circuit. IEEE Microwave Wireless Components Letters, 2011, vol. 21, no. 1, p. 19-21. DOI: 10.1109/LMWC.2010.2089675

\section{About the Authors ...}

Te SHAO was born in Harbin, China. He received his B.Eng. and M.Eng. degrees in Information and Communication Engineering from Dalian Maritime University, Liaoning, China, in 2013 and 2016. His research interests include negative group delay circuit and optimization method.
Shaojun FANG received the Ph.D. degree in Communication and Information Systems from Dalian Maritime University (DLMU), Liaoning, China, in 2001. Since 1982, he has been with DLMU, where he is currently the Head Professor with the School of Information Science and Technology. His recent research interests include passive RF components, patch antennas, and computational electromagnetics. He has authored or co-authored three books and over 100 journal and conference papers. He was a recipient of the Best Doctor's Dissertation Award of Liaoning Province in 2002 and the Outstanding Teacher Award of the Ministry of Transport of China.

Zhongbao WANG (corresponding author) received the Ph.D. degree in Communication and Information Systems from Dalian Maritime University (DLMU), Liaoning, China, in 2012. He is currently an Associate Professor with the School of Information Science and Technology, DLMU. His current research interests include passive RF components, patch antennas, and microwave technology using artificial intelligence. He has authored or co-authored over 50 papers in journals and conferences. He is currently serving as a Technical Reviewer for the IEEE Trans. on MTT, IEEE AWPL, IET MAP, Electronics Letters, Radioengineering, ETRI Journal, and International Journal of RF and Microwave Computer-Aided Engineering. He was a recipient of the Best Doctor's Dissertation Award of Liaoning Province in 2013.

Hongmei LIU received the Ph.D. degree in Information and Communication Engineering from Dalian Maritime University (DLMU), Liaoning, China, in 2016. She is currently a Lecturer with the School of Information Science and Technology, DLMU. Her current research interests include passive microwave circuits, reconfigurable RF components, and CP microwave antennas. 